\title{
THE LIGHT CURVE OF THE SYMBIOTIC NOVA HM SAGITTAE
}

\author{
V.P. Arkhipova, T.S. Belyakina, O.D. Dokuchaeva, \\ and R.I. Noskova
}

Sternberg State Astronomical Institute, Moscow, U.S.S.R.

The light curve of the symbiotic nova HM Sge in 1960-1988 derived from 260 photographic and 243 photoelectric observations (Arkhipova et al. 1989) in presented in Fig. 1.

The rate of nova-like brightness increase in 1975 was about 0.04 mag/day. The maximum duration was about 4 years, with brightness oscillations of up to $1 \mathrm{mag}$. After 1981 the star brightness decreased slowly (at the rate of $\sim 0.08 \mathrm{mag} / \mathrm{yr}$ ) with small oscillations of 0.2 $\mathrm{mag}$ in the $\mathrm{B}$ band.

Fourteen years before the outburst of HM Sge a preoutburst phase with a light amplitude of $1 \mathrm{mag}$ and a duration of about two years was observed, but its reality is rather doubtful. similar features can also be seen in the light curves of the symbiotic novae V1016 Cyg and V1329 Cyg, 15 and 12 years before their outbursts, respectively.

Fig. 2 shows the mean, strongly smoothed, light curves of RR Tel, V1016 Cyg, V1329 Cyg and HM Sge shifted in the time axis as to coincide at the time of the rise to maximum. The light curve of the classical slow nova HR Del with maximum brightness duration of 300 days is shown for comparison.

The red components in RR Tel, V1016 Cyg and HM Sge have been found to be Mira stars. Before the outburst a Mira component was observed only in RR Tel (FitzGerald et al. 1966), while after the outburst it was discovered in HM Sge and V1016 Cyg from infrared observations. The period of the Mira in HM Sge is about 500 days (Taranova and Yudin 1983). In the optical region of HM Sge, a period of about 550 days may be present, as shown in Figure 3. The data are from our post-outburst UBV observations. Its origin is vague, but this may be due to some oscillation of the integral brightness of the binary modulated by the Mira.

As it clearly appears from Fig. 2, the photometric parameters of symbiotic novae in outbursts such as the outburst amplitude, the maximum absolute magnitude, the photometric behaviour at maximum and afterwards, are different from system to system. In our opinion, these differences are caused by two factors: a) differences in the optical depth of the dust circumstellar envelopes around the cold components (more in HM Sge, less in RR Tel) and, b) differences in the optical depth of the expanding gaseous envelopes around the hot components. The high optical depth of the gaseous envelope of RR Tel in the continuum and in the emission lines is responsible for the absorption spectrum of the supergiant at maximum, which is typical for all classical novae during maximum and early decline. The optical depth for HM Sge and V1016 Cyg at maximum was substantially less, which explains their lower optical luminosity, the lack of an absorption line spectrum and the very flat and long maximum. The light maxima of HM Sge, V1016 Cyg and probably also V1329 Cyg, coincide in the main characteristics with the early nebular stages of novae. The preceeding stages, always present in classical novae, are not observed in symbiotic novae. 
If we assume the present model of nova outburst as a runaway at the surface of an accreting white dwarf, then the photometric behaviour of classical and symbiotic novae may be ascribed to the different rates of energy release during the star outburst, as well as to the difference in mass and expansion velocity of the envelope ejected by the white dwarf.

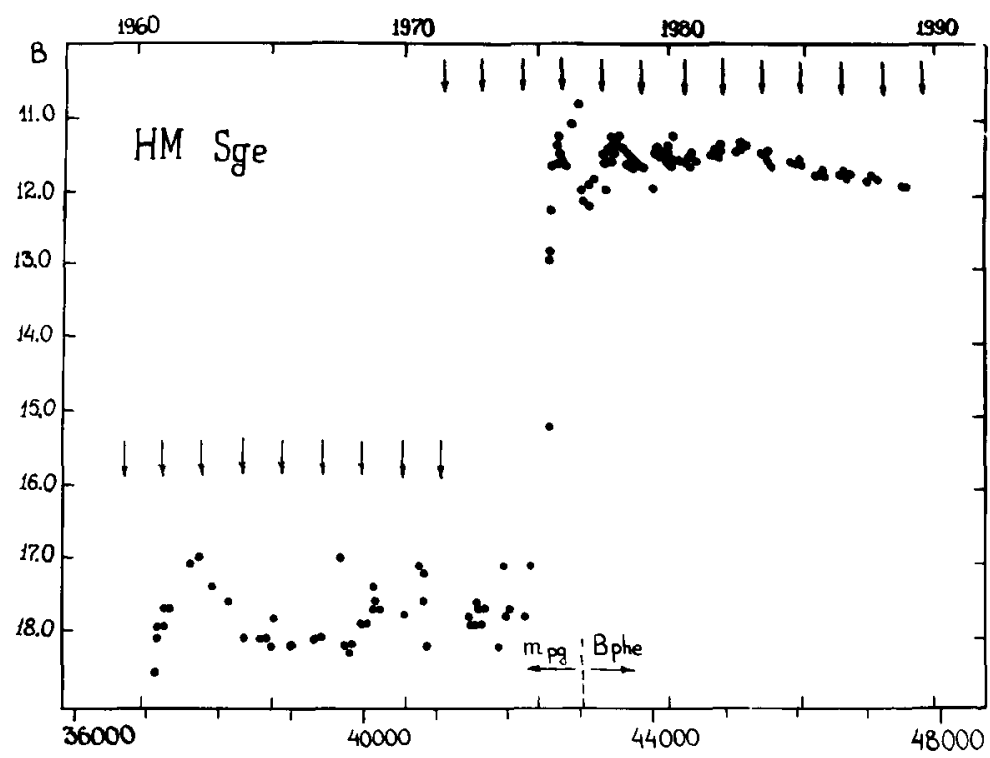

Fig. 1: Light curve of HM Sagittae in 1960-1988. Photographic magnitudes are given before Julian Date 43050. After this date, the measurements refer to photoelectric $B$ magnitudes. The data were averaged in 20 days intervals. The arrows show the light minima according to: $\operatorname{Min}(B)=45000-550 \mathrm{E}$.

\section{REFERENCES}

Arkhipova, V.P., Belyakina, T.S., Dokuchaeva, O.D., Noskova, R.I.: 1989, Izv. Krimsk. Astrofis. Obs. Vol. 78, p. 41

FitzGerald, M.P., Houk, N., McCuskey, S.w., Hoffleit, D.: 1966 : Astrophys. J. 144, 1135

Taranova, O.G., Yudin, B.F.: 1983, Astron. Astrophys., 117, 209 


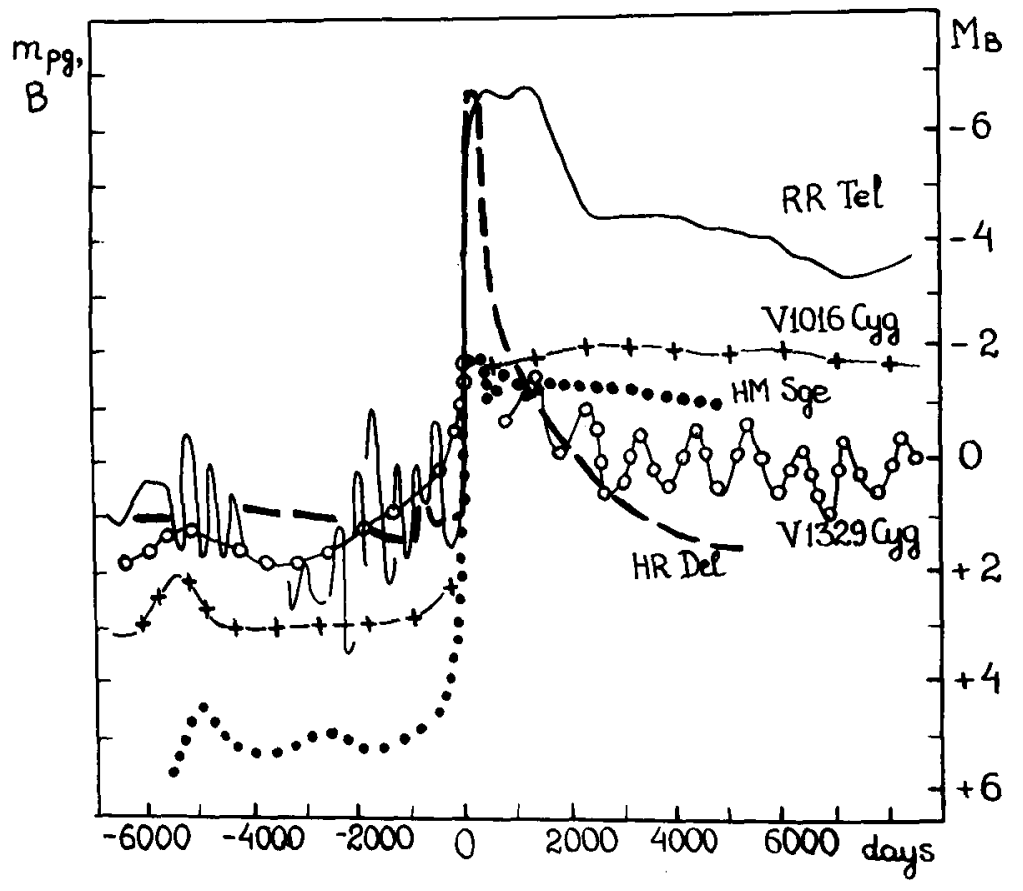

Fig. 2: Comparison of the blue light curves of the symbiotic novae RR Tel, V1016 Cyg, V1329 Cyg, and HM Sge shifted in time to coincide at the time of rise to maximum. The light oscillations are somewhat smoothed. The curves are shifted along the ordinate axis to keep the relation of the absolute magnitudes in maximum light.

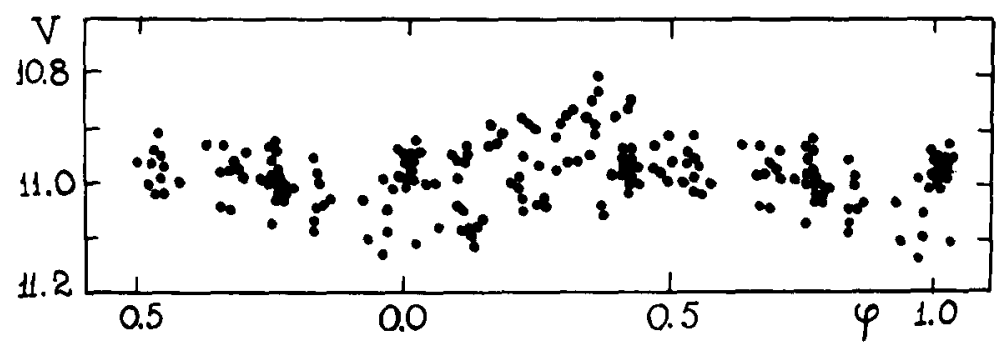

Fig. 3: Post-outburst observations of HM Sge reduced with a period of 550 days. The brightness trend was taken into account as $V_{c o o r}=V-$ 0.00017 (JD - 45150). For JD< 45150 the trend was not accounted for. 